\title{
A rare case of extraskeletal pulmonary myxoid chondrosarcoma
}

\author{
Kendal M. Endicott, Stephanie Barak, Keith D. Mortman* \\ The George Washington University Hospital, United States
}

Received: September 15, 2015

DOI: $10.5430 /$ jst.v6n1p18
Accepted: November 10, $2015 \quad$ Online Published: November 23, 2015

URL: http://dx.doi.org/10.5430/jst.v6n1p18

\begin{abstract}
Primary malignant cartilaginous tumors of the lung are rare with limited reports in the literature. Here we describe the case of a 69-year-old male who presented with a lobulated left upper lobe mass. Preoperative percutaneous biopsy was consistent with a mucinous adenocarcinoma. Final pathology demonstrated a primary extraskeletal pulmonary myxoid chrondrosarcoma. The clinical presentation and histologic characteristics are described.
\end{abstract}

Key Words: Thoracoscopy/VATS, Lung pathology, Sarcoma (lung)

\section{INTRODUCTION}

Primary pulmonary malignancies are most commonly of epithelial origin. Tumors of other histologic differentiation have been described in association with the airway and bronchi, however primary tumors with cartilaginous or osteoid features originating in the lung are extremely rare and typically indicate metastatic disease. While cases of extraskeletal myxoid chondrosarcoma of the extremities are extremely infrequent, descriptions of this tumor histology arising from the lung are isolated to case reports in the literature. Here we review a case of a suspected pulmonary adenocarcinoma by preoperative biopsy with final pathology demonstrating an extraskeletal pulmonary myxoid chondrosarcoma.

\section{Case presentation}

A 69-year-old male presented to the Emergency Department with acute onset of dyspnea. His past medical history was significant for hypertension and hyperlipidemia, and he had a 100 pack-year smoking history with ongoing tobacco use. Computed tomography (CT) of the chest was performed to rule out a pulmonary embolism (PE). No PE was found, however the $\mathrm{CT}$ demonstrated a $4.8 \mathrm{~cm} \times 2.5 \mathrm{~cm}$ lobulated mass in the posterior segment of the left upper lobe (see Figure 1).

No additional masses, lymphadenopathy, or effusions were present. The patient subsequently underwent positron emission tomography-CT (PET-CT) which demonstrated hypermetabolic activity in the left upper lung mass with a standard uptake value (SUV) of 9.0. There were no other hypermetabolic foci. Brain magnetic resonance imaging (MRI) was negative for metastatic disease. Bone scan was positive at the right eighth rib and right frontal calvarium. The eighth rib focus was biopsied and negative for malignancy. The lesion on the calvarium corresponded to an inflammatory focus on the patient's scalp. Preoperative CT-guided biopsy of the left upper lobe mass at another institution demonstrated mucinous adenocarcinoma. The histology was reviewed at a second outside institution. At the time of presentation to our clinic, he was asymptomatic. Vital signs were within normal limits and room air pulse oximetry was $96 \%$. His cardiac and pulmonary examination was normal and there was no palpable adenopathy. There was a 4 month interval between

*Correspondence: Keith D. Mortman; Email: kmortman@mfa.gwu.edu; Address: The George Washington University Hospital, United States. 
his initial CT scan and thoracic surgery referral. A repeat CT scan showed enlargement of the left upper lobe mass to $6.8 \mathrm{~cm} \times 4.6 \mathrm{~cm}$. There were no new masses or adenopathy. Preoperative pulmonary function tests were significant for a forced expiratory volume in 1 second (FEV1) of 2.55 liters (61\% predicted) and diffusion capacity of carbon monoxide (DLCO) $71 \%$ of predicted. A staging mediastinoscopy was negative. During left thoracoscopy, there were no adhesions between the left upper lobe and chest wall. Despite reasonable preoperative PFTs, the patient experienced significant difficulties with oxygenation while on single lung ventilation. Therefore a sublobar resection was performed to quickly restore two lung ventilation, improve oxygenation, and preserve parenchyma. Postoperatively, the patient did well and was discharged home on the first postoperative day.
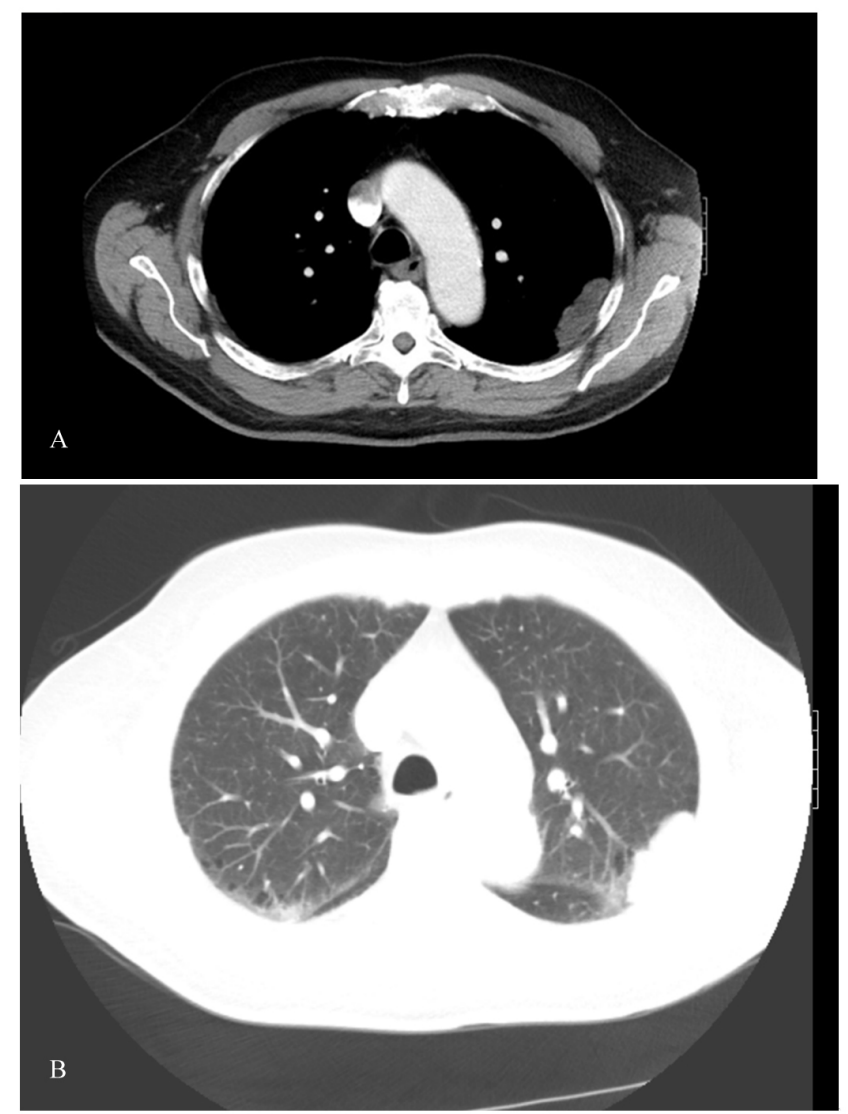

Figure 1. Axial CT scan of left upper lobe mass, mediastinal window (Panel A) and lung window (Panel B).

Gross examination revealed a $4.8 \mathrm{~cm} \times 3.5 \mathrm{~cm} \times 3.0 \mathrm{~cm}$ ill-defined mass with a fleshy, mucinous cut surface. The parenchymal margin appeared uninvolved, however the tumor was extending to the pleural surface with puckering.

Microscopic examination showed a well-demarcated, multilobulated mass with fibrous septa and atypical epithelioid cells with round to oval nuclei, moderate nuclear atypia arPublished by Sciedu Press ranged in cords, nests and trabeculae in a prominent myxoid matrix (see Figure 2).

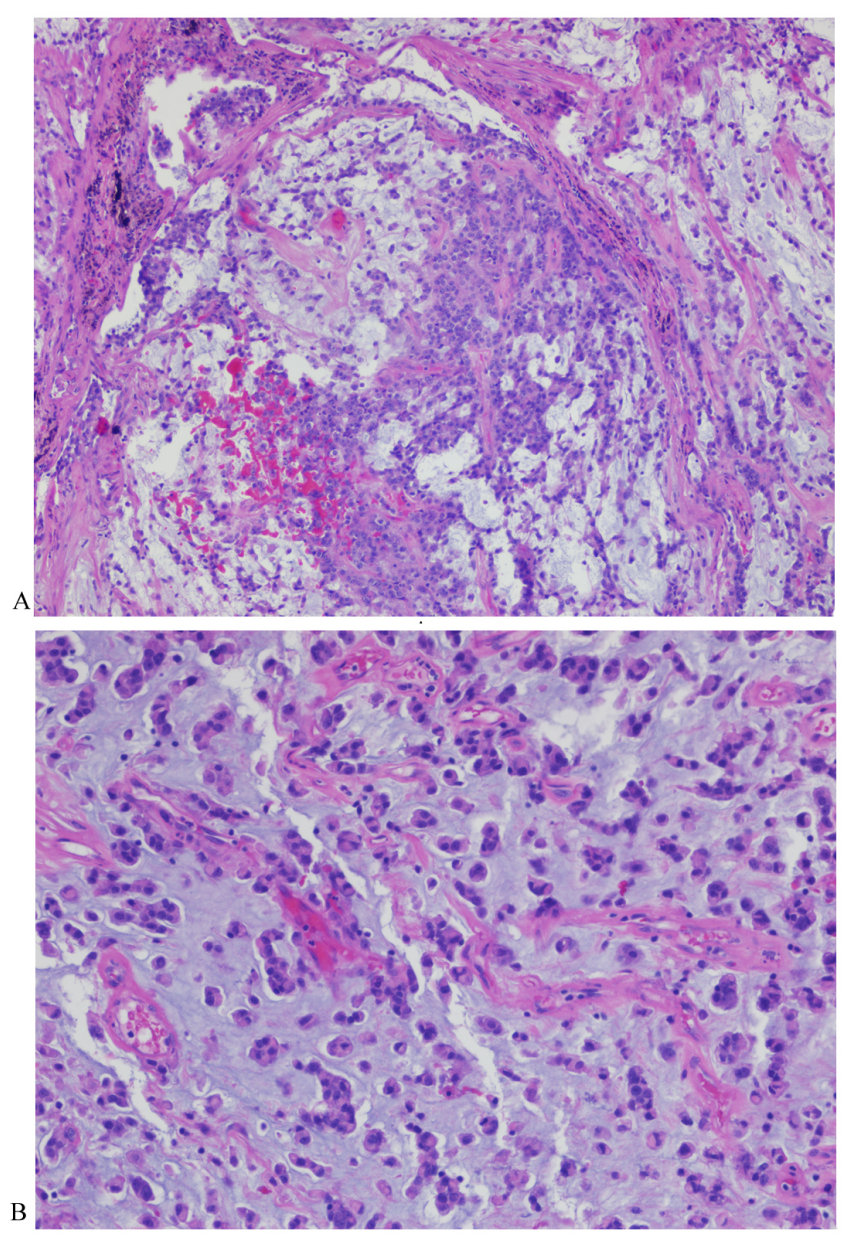

Figure 2. Low power magnification $(10 \times)$ : multilobulated mass with fibrous septa and myxoid background (Panel A). High power magnification $(20 \times)$ : atypical epithelioid cells with round to oval nuclei, moderate nuclear atypia arranged in cords, nests and trabecula in a prominent myxoid matrix (Panel B).

Focal tumor necrosis was present, and mitotic figures were frequent (16 MF/HPF). By immunohistochemistry, the cells were positive for synaptophysin and focally positive for $\mathrm{S} 100$. AE1/AE3, calretinin, CD68, CK5/6, CK7, CK20, desmin, EMA, MAK6, MOC31, myoglobin, Napsin A, and TTF-1 were negative in the tumor cells. These findings were most consistent with an extraskeletal pulmonary myxoid chondrosarcoma. EWSR1 rearrangement studies were negative. Concurrent staging revealed no metastatic disease in the mediastinal lymph nodes.

At his 6 month postoperative visit, he was doing well with no clinical or radiographic evidence of recurrence. 


\section{Discussion}

Extraskeletal myxoid chondrosarcoma (EMC) are sarcomas usually involving muscles or subcutis. The common location is the deep thigh, however EMC can be found in the hand, retroperitoneum, and head and neck. On CT imaging, chondrosarcomas often appear as large soft tissue masses with heterogenous and intratumoral calcification with occasional destruction of the adjacent bone. ${ }^{[1]}$

EMC has a classic histologic appearance with multiple myxoid lobules, separated by fibrous septa, and tumor cells arranged in cord or strands in a netlike or linear interconnected pattern like a "string of pearl". [2] Immunohistochemically, most EMC are positive for vimentin and focally positive for S100. They are negative for cytokeratin (except EMA) and GFAP. Cytogenetic molceular studies have shown that EMC is associated with a reciprocal translocation $\mathrm{t}(9 ; 22)$ ( $\mathrm{q} 22$ ; q12), generating an EWS/NR4A3 gene fusion in at least $75 \%$ of the cases. To date, there is no clear understanding of the correlation between presence or absence of the gene rearrangement and tumor behavior. ${ }^{[3]}$ On resection specimens, the differential diagnosis is limited, and it is important to exlude metastasis from a soft tissue EMC.

Primary chondrosarcomas of the lung are extremely rare with only isolated case reports found in the literature. The largest series reported by Kalhor et al. highlights four cases-two of myxoid character and two hyaline type. ${ }^{[4]}$ In 2012, two case reports were published describing one $\mathrm{EMC}^{[5]}$ in a 51year old female with severe anemia and primary pulmonary myxoid sarcoma with EWSR1-CREB1 fusion, resembling EMC. ${ }^{[3]}$ Matsukuma et al. highlight the difficulty classifying myxoid sarcoma arising from the lung and recommend characterizing them as "primary pulmonary myxoid sarcoma".
Given the rarity of this tumor, statements regarding treatment and further prognosis of the described patient can only be extrapolated from other cartilaginous tumor types. The biologic activity of chondrosarcomas is widely variable and generally correlates with tumor histology. EMC is typically regarded as a low grade chondrosarcoma; however, more recent studies have demonstrated high potential for metastasis and local recurrence suggesting a more intermediate malignancy of this tumor. ${ }^{[6]}$ Kawaguchi et al. present data to support the role of a wide local excision due to a high rate of local recurrence. ${ }^{[7]}$

Chondrosarcomas are notoriously resistant to conventional chemotherapy and radiation treatments, making curative resection the only definitive cure. In advanced or metastatic tumors, recent studies with novel chemotherapy regimens have not demonstrated increased survival. ${ }^{[8]}$ Further efforts are needed in this area to define a more effective strategy. The overall prognosis of ECM is difficult to estimate given the rarity of the tumor, but data from low-grade chondrosarcomas reports a 10-year survival rate between $80 \%-90 \%$. Highgrade chondrosarcomas have a worse prognosis with10-year survival estimated between $40 \%-70 \% .{ }^{[9]}$ The origin of chondrosarcoma does seem to correlate with prognosis, however it is unclear if this association is due to unfavorable pathology or the inability to perform an $\mathrm{R} 0$ resection for many tumors.

Given the poor response of chrondrosarcomas to chemotherapy and radiation, no further therapy is planned in our patient. Routine surveillance CT imaging will be performed at 6 month intervals.

\section{CONFLicts OF InTEREST Disclosure}

The author declares that there is no conflict of interest statement.

\section{REFERENCES}

[1] Wang XY, Hu S, Guo LC, et al. Primary chondrosarcoma presenting as an intrathoracic mass: a report of three cases. Oncol Lett. 2014; 8(3): 1151-4. http://dx.doi.org/10.3892/ol.2014.2275

[2] Miettinen. Modern Soft Tissue Pathology. Chapter 30. Cambridge University Press. 2010: 867-9.

[3] Matsukuma S, Hisaoka M, Obara K, et al. Primary pulmonary myxoid sarcoma with EWSR1-CRB1 fusion, resembling extraskeletal myxoid chondrosarcoma: Case report with review of literature. Pathol Int. 2012; 62: 817-22. PMid:23252871. http://dx.doi .org/10. $1111 /$ pin. 12014

[4] Kalhor N, Suster S, Moran CA, et al. Primary pulmonary chrondrosarcomas: a clincopathologic study of 4 cases. Hum Pathol. 2011; 42: 1629-34. PMid:21496864. http://dx.doi.org/10.1016/j .humpath.2011.01.009

[5] Zhou Q, Lu G, Liu A, et al. Extraskeletal myxoid chondrosarcoma in the lung. Asymptomatic lung mass with severe anemia. Diagn Pathol.
2012; 7: 112. PMid:22925697. http://dx.doi.org/10.1186/1 746-1596-7-112

[6] Kinoshita T, Kamiyama I, Hayashi Y, et al. Spontaneous regression of metastatic extraskeletal myxoid chondrosarcoma. Ann Thorac Surg. 2015; 100(4): 1465-7.

[7] Kawaguchi S, Wada T, Nagoya S, et al. Extraskeletal myxoid chondrosarcoma: a multi-institutional study of 42 cases in Japan. Cancer. 2003; 97: 1285-92. PMid:12599237. http://dx.doi.org/10.10 $02 /$ cncr. 11162

[8] van Oosterwijk JG, Herpers B, Meijer D, et al. Restoration of chemosensitivity for doxorubicin and cisplatin in chondrosarcoma in vitro: BCL-2 family members cause chemoresistance. Ann Oncol. 2012; 23: 1617-26. PMid:22112972. http://dx.doi .org/10.10 93/annonc/mdr512

[9] SRoos E, van Coevorden F, Verhoef C, et al. Prognosis of primary and recurrent chondrosarcoma of the rib. Ann Surg Oncol. Advance online publication. 